\title{
PRESSURE FIELD STUDY OF THE TEVATRON COLD COMPRESSORS
}

\author{
A. L. Klebaner, A. Martinez, W. M. Soyars and J. C. Theilacker \\ Fermi National Accelerator Laboratory \\ Batavia, IL, 60510, USA
}

\begin{abstract}
The Fermilab Tevatron cryogenic system utilizes high-speed centrifugal cold compressors, manufactured by Ishikawajima-Harima Heavy Industries Co. Ltd. (IHI), for high-energy operations [1]. The compressor is designed to pump $60 \mathrm{~g} / \mathrm{sec}$ of $3.6 \mathrm{~K}$ saturated helium vapor at a pressure ratio of 2.8, with an off-design range of 40 to $70 \mathrm{~g} / \mathrm{sec}$. Operating speeds are between 40 and $95 \mathrm{krpm}$, with a speed of $80 \mathrm{krpm}$ at the design point. Different heat loads and magnet quench performance of each of the twenty-four satellite refrigerators dictates different process pressure and flow rates of the cold compressors. Reducing the process flow rate can cause the centrifugal cold compressor to stop pumping and subsequently surge. Tests have been conducted at the Cryogenic Test Facility at Fermilab to map the pressure field and appropriate efficiency of the IHI hydrodynamic cold compressor. The information allows tuning of each of the twenty-four Tevatron satellite refrigerators to avoid cold compressor operation near the surge and choke lines. A new impeller has also been tested. The Tevatron cold compressor pressure field and efficiency data with the new impeller are presented in this paper.
\end{abstract}

\section{INTRODUCTION}

The Tevatron superconducting magnets are cooled by a hybrid cryogenic system, which consists of a Central Helium Liquefier (CHL) and 24 satellite refrigerators. Each of the satellite refrigerators cools two $125 \mathrm{~m}$ magnet strings. The magnets are of warm iron design. Lower operating temperatures allow the magnets to carry higher current without quenching, and consequently increase the energy of the particle beam in the accelerator.

A single stage cold compressor is used in each of the 24 satellite refrigerators to lower the operating temperature of the Tevatron accelerator. The primary machine is a high-speed centrifugal compressor supplied by IHI. A shroudless, cast aluminum impeller is driven by a $1.5 \mathrm{~kW}$ induction motor. The vertical rotor assembly consists of a steel shaft and squirrel cage type rotor. The assembly is supported radially by two identical self-acting foil gas bearings and axially by a spiral groove gas thrust bearing. 
Unique quench characteristics of individual magnets and variation in static heat load in each of the twenty four satellite refrigerators dictates different process pressure and flow rates of the cold compressors. Currently, the inlet pressure of the Tevatron cold compressors varies from 55 to $77 \mathrm{kPa}$, where steady state operating speed (N) is in the range of 50 to $80 \mathrm{krpm}$.

We have experienced multiple machine trips in reduced flow regimes such as transient system modes during quench recovery. Historically, cold compressors operating at lower inlet pressures and higher speeds have been especially susceptible to these machine trips. A program of testing cold compressors was begun to determine the pressure field and appropriate efficiency of the machines. Tests were conducted at the Cryogenic Test Facility at Fermilab. The information collected allow for better tuning of each of the twenty-four satellite refrigerators to avoid cold compressor operation near these forbidden (surge or choke) regions.

\section{TEST SETUP}

FIGURE 1 illustrates a setup configuration for the IHI cold compressor tests. Two Tevatron style helium refrigerators with nominal capacity of $600 \mathrm{~W}$ at $4.5 \mathrm{~K}$ are utilized for the test. In FIGURE 1, P, T, F, H and L stands for pressure transducer, temperature sensor, flow meter, heater element and liquid level probe, respectively.

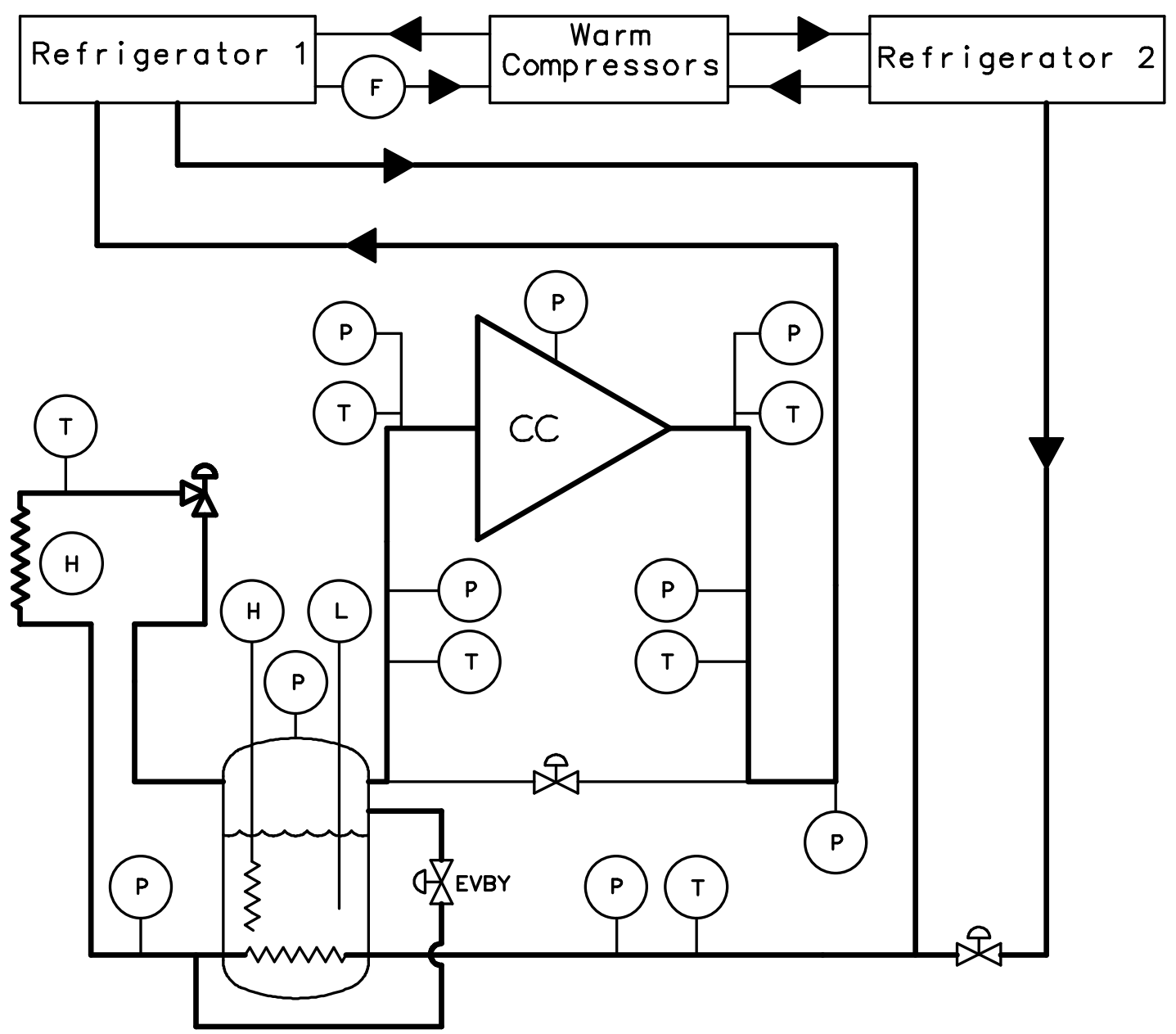

FIGURE 1. Simplified flow schematic of the cold compressor test set-up. 
Refrigerator \#1 is configured to run in a satellite mode, where Refrigerator \#2 is in liquefier mode, emulating Fermilab's Central Helium Liquefier (CHL). A standalone valve box, which closely matches the standard Tevatron valve box in almost every detail, is used as a centerpiece of the distribution system. All of the components are connected via vacuum insulated transfer tubes. The electrical heater acts as a load to the refrigerators to allow for a fine system control during transient conditions. The cold compressor cryostat was modified to add instrumentation (thermometers and pressure probes) as close to the impeller/shroud as practical.

In addition to the existing standard Tevatron refrigerator instrumentation, calibrated Lakeshore Cryotronics CERNOX temperature resistors, model number CX-1050-SD, as well as SETRA and MKS pressure sensors were installed at points of interest in the test setup. To measure cold compressor flow rate, a factory calibrated FCI model LT81A thermal flowmeter was installed on the low-pressure header between Refrigerator \#1 and warm compressor suction.

All of the instruments have been lab calibrated and field verified. Temperature resistors where flooded in two-phase helium and compared against saturation pressure that was read by corresponding pressure sensors. Pressure transducers were checked against a high precision reference instrument. The thermal flowmeter was checked against an orifice style flow meter in the system.

The controls and data acquisition system are based on Siemens-Moore Industrial Advanced Process Automation and Controls System (APACS). The APACS system is interconnected with the Fermilab Accelerator Network (ACNET) via a Data Acquisition Engine (DAE) developed by Fermilab Beams Division Controls Department personnel. An ACNET datalogger is used for storing, displaying and retrieving of the test data.

\section{TEST RESULTS}

Two series of tests were conducted - one with the original IHI impeller, another with an alternative impeller designed and manufactured by Turbotechnology Services Corporation (TSC). No significant differences in machine performance and operating limits can be distinguished between the two series. For simplicity, only data collected from the compressor tests with the TSC impeller are presented in TABLE 1.

TABLE 1. Fermilab's cold compressor surge parameters

\begin{tabular}{|c|c|c|c|c|c|}
\hline & \multicolumn{5}{|c|}{ Surge Point } \\
\cline { 2 - 5 } Run \# & Speed $[\mathbf{k r p m}]$ & $\begin{array}{c}\text { Suction } \\
\text { Pressure }[\mathbf{k P a}]\end{array}$ & $\begin{array}{c}\text { Discharge } \\
\text { Pressure } \\
{[\mathbf{k P a}]}\end{array}$ & $\begin{array}{c}\text { Temperature } \\
{[\mathbf{K}]}\end{array}$ & $\begin{array}{c}\text { Mass Flow } \\
{[\mathbf{k g} / \mathbf{s e c}]}\end{array}$ \\
\hline 1 & 44 & 75 & 111 & 5.394 & 0.016 \\
2 & 49 & 70 & 115 & 5.241 & 0.027 \\
3 & 54 & 67 & 119 & 5.459 & 0.029 \\
4 & 59 & 61 & 118 & 5.648 & 0.028 \\
5 & 64 & 56 & 119 & 5.849 & 0.030 \\
6 & 69 & 52 & 120 & 6.069 & 0.029 \\
7 & 74 & 47 & 119 & 6.356 & 0.028 \\
8 & 79 & 43 & 118 & 6.608 & 0.029 \\
9 & 84 & 47 & 140 & 6.587 & 0.052 \\
10 & 89 & 44 & 142 & 6.793 & 0.055 \\
\hline
\end{tabular}




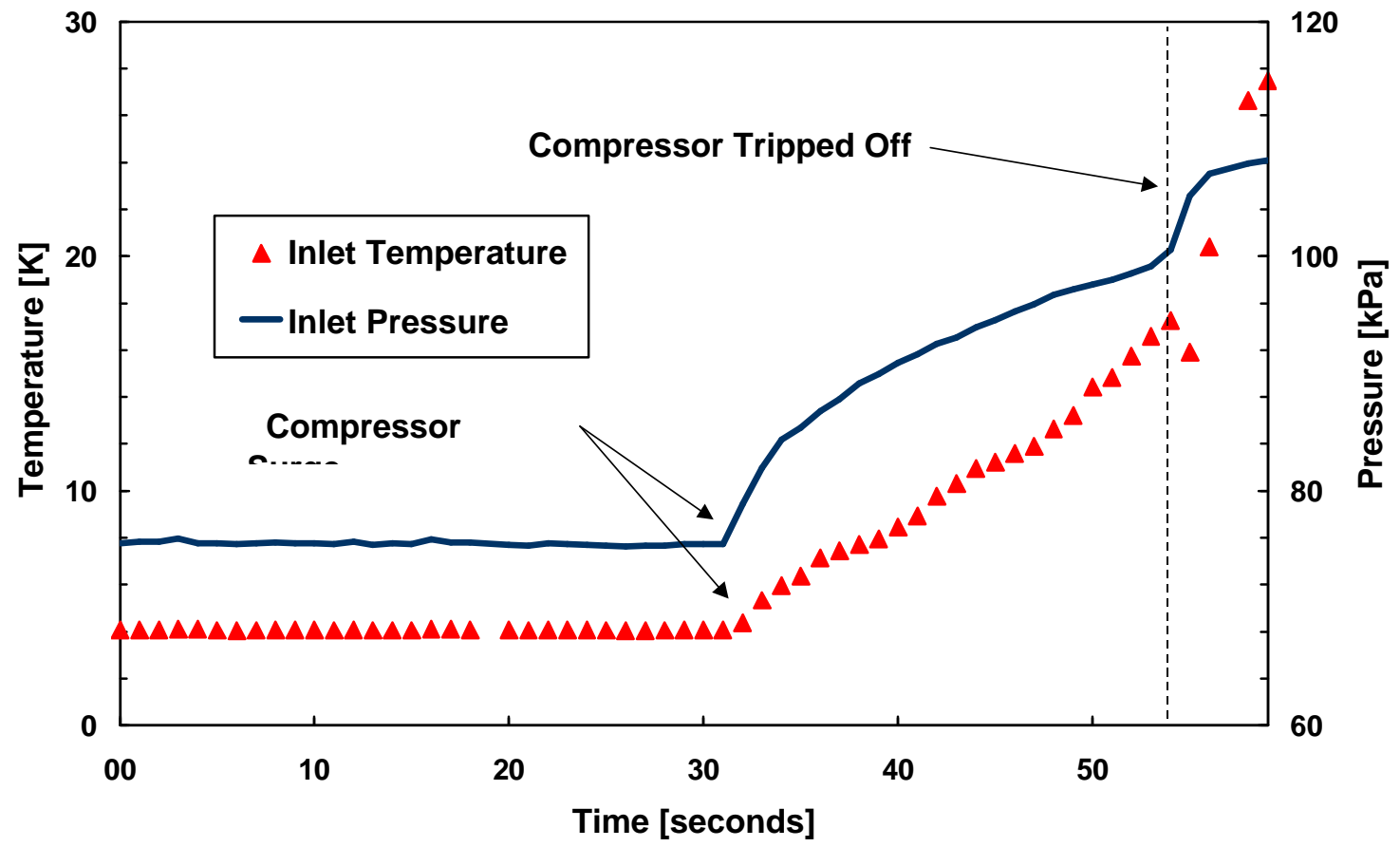

FIGURE 2. A typical behavior of cold compressor during surge.

Each series of tests consisted of ten runs. During each run the cold compressor speed was locked at a fixed value, while the flow rate was varied using bypass valve (EVBY) .

Cold compressor vibration was continuously monitored. We obtained vibration information when the compressor moved into the surge regime to see if vibrations increased significantly in these areas. Furthermore, we were concerned with the rotorbearing system's $2^{\text {nd }}$ critical speed resonance, which can be close to the compressor's minimum operating speed [2]. An accelerometer was mounted to the exterior of the cryostat in the radial direction, a location found to have the highest sensitivity. Sample rates of up to $1 \mathrm{MHz}$ were used to monitor the signal, which has a frequency on the order of the rotational speed. The accelerometer time-domain signal was observed. The oscilloscope's fast Fourier transform capabilities converted this into the spectral frequency components.

Limited by the refrigeration capacity of the system and the maximum range of the thermal flowmeter, we were unable to reach the choke line - the line characterized by sonic velocity in some section of the machine, thus further increase of flow is not possible. On the other hand, surge, or severe oscillations of the mass flow rate through the machine, has been observed during each run.

As shown on FIGURE 2, when reaching the surge point, a sudden increase in inlet temperature and pressure was observed. This is due to flow recompression following flow reversal in the impeller wheel. In some cases, after approximately 30 seconds, the system would recover and the surge characteristics would repeat. This cycle would occur repeatedly unless the flowrate was increased to force the compressor out of the surge regime. If the flowrate is kept unchanged, the machine will eventually trip off. 


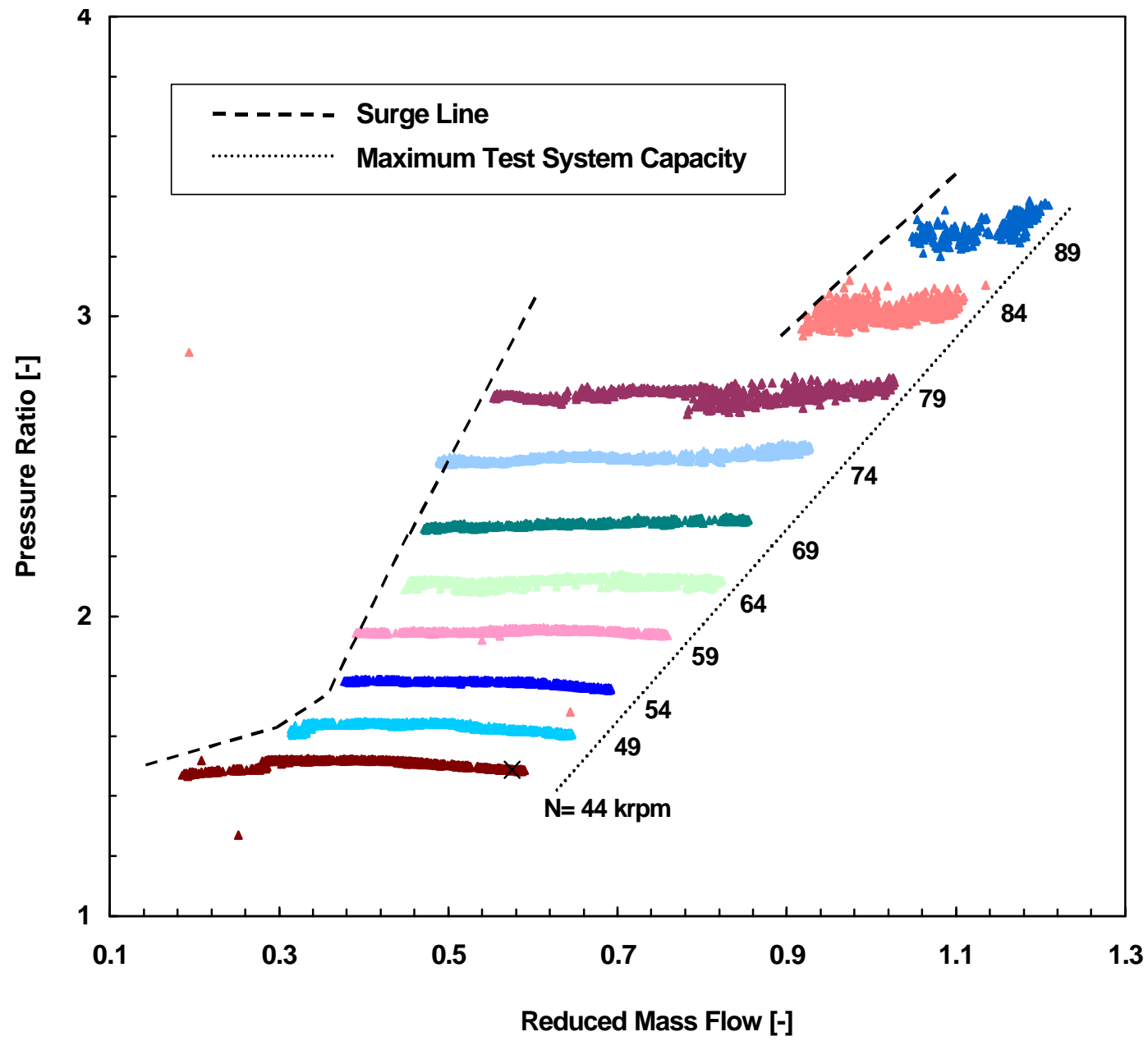

FIGURE 3. The Fermilab cold compressor hydrodynamic pressure field.

No significant changes in vibration readings were observed leading up to the surge. Based on industry standards and recommendations for the compressor's operating conditions, we desire casing accelerations <0.3 g. [3]. We observed readings in the acceptable range, 0.1-0.3 g. These readings are in agreement with those from earlier rotordynamic testing [2].

For the two compressor configurations tested, we saw no evidence of increase mechanical response as the machine approached or reached the surge point. We did not conclusively identify a critical speed response in the machines' operating range since no specific frequency producing large resonance was observed.

The mapping results are presented in FIGURE 3. The map is presented in traditional dimensionless coordinates - pressure ratio versus reduced mass flow rate [4-5]. The pressure ratio is a ratio of the outlet pressure to inlet pressure, while reduced mass flow is the actual mass flow through the machine scaled by the inlet conditions and referenced to the design point parameters [4].

For the machine under discussion, at a constant speed the reduced mass flowrate has a weak dependence on compressor pressure ratio. The dependence begins to increase at the extremes of speed range. The majority of the plotted data is on the left side of the constant speed curve's maximum. For the low speed range, up to $64 \mathrm{krpm}$, a shallow maximum can be seen. The $69 \mathrm{krpm}$ line is almost perfectly parallel to the abscissa. At speeds higher than $69 \mathrm{krpm}$, we were unable to reach the peak due to test system limitation. 


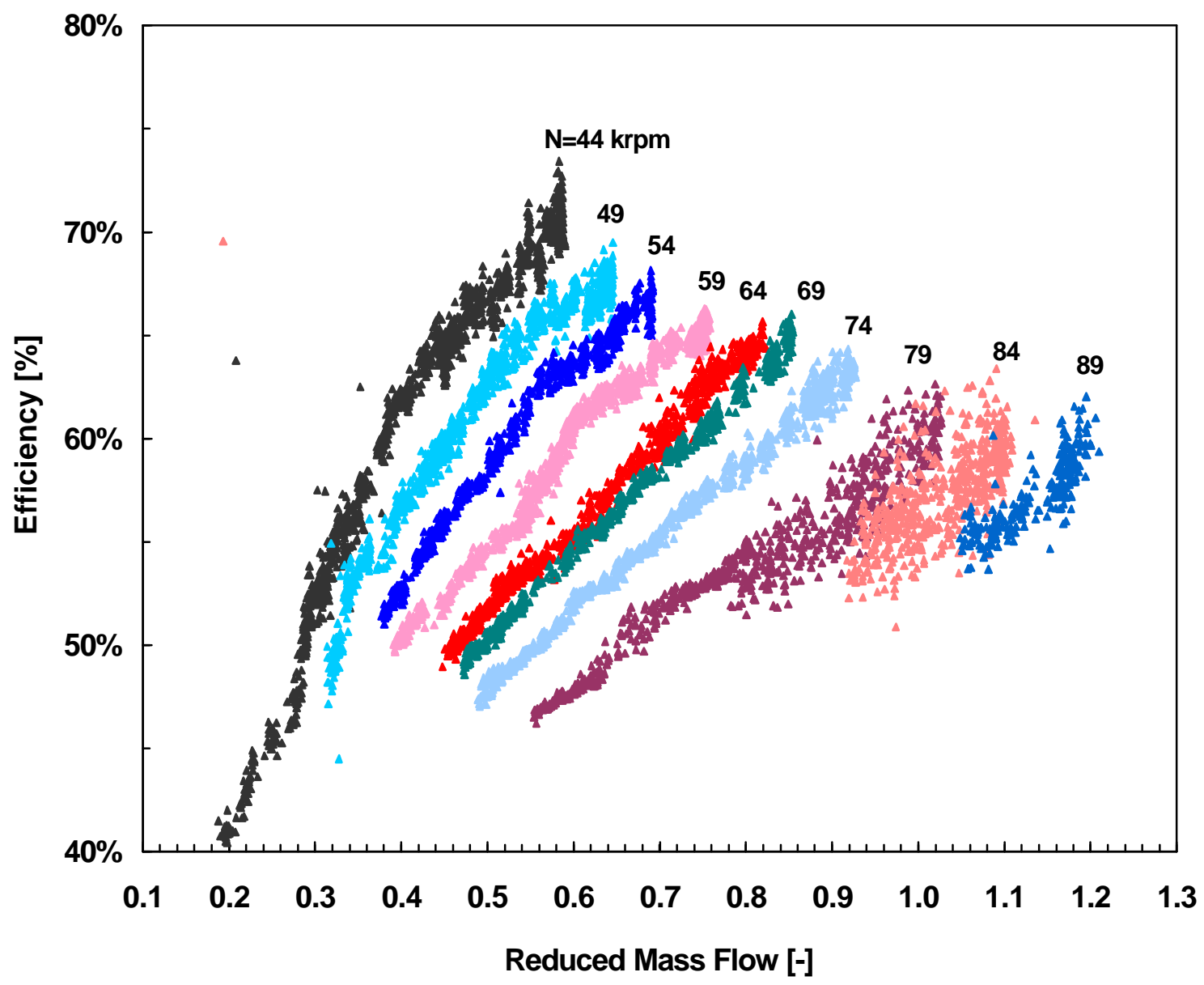

FIGURE 4. The Fermilab cold compressor hydrodynamic pressure field.

An important observation is that at speeds above $79 \mathrm{krpm}$, the cold compressor enters a region of increased fluctuations in inlet and outlet pressure as well as inlet and outlet temperatures. At speeds above $79 \mathrm{krpm}$, the surge line is also reached at a much higher flowrate than would be anticipated based on lower speed mapping runs. The cause of the fluctuations and discontinuity is not understood at this time.

The adiabatic efficiency as a function of the reduced mass flow rate for a speed range from $44 \mathrm{krpm}$ to $89 \mathrm{krpm}$ is presented in FIGURE 4. At slower speed, the efficiency of the cold compressor becomes a stronger function of the reduced mass flowrate. This, coupled with the fact that for this machine the pressure ratio is almost independent from the reduced mass flowrate at a constant speed, suggests that the dominant loss is due to helium leakage across the impeller as opposed to friction losses.

The operational experience of twenty-four compressors in the Tevatron showed that the average compressor adiabatic efficiency was lower than the prototype machine [6]. This was suspected to be due to pressure drop across the compressor's inlet bayonet. To investigate this, additional pressure transducers within the test cryostat were installed. Tests have shown the bayonet pressure drop to be low. The compressor pressure field and efficiency map now suggests that the change in efficiency is a result of lower compressor mass flows during Tevatron operations, than those used during prototype testing.

An unexpected result of these tests was the observation that the cold compressor housing pressure would go subatmospheric at compressor inlet pressures below $76 \mathrm{kPa}$. The motor housing is filled with stagnant ambient temperature helium, which 
communicates with the discharge side of the impeller. The higher the compressor mass flow, the lower the housing pressure. This suggests that gas leaving the impeller discharge ejects gas from the motor housing. The housing is sealed to atmosphere via a single O-ring and is susceptible to air inleak if the housing pressure is subatmospheric. If a leak occurs, contaminants will migrate to cold surfaces of the machine. This may have contributed to locked rotor conditions that were observed in the Tevatron since the low temperature upgrade [7]. The potential of contaminants migrating from motor housing down to the cold end prompted us to initiate a rigorous procedure of leak checking the joints and seals, followed by pumping and backfilling during a modest heating of the housing.

\section{SUMMARY}

Tests were performed to map the hydrodynamic parameters and efficiencies of the Fermilab's centrifugal cold compressor. Surge parameters for the machines' operating range were identified. Compressors efficiency was found to vary over a wider range than anticipated, especially at lower speeds. The information collected during the tests allows for improved tuning of each of the twenty-four Tevatron satellite refrigerators to avoid cold compressor operation near unstable regions.

The hydrodynamic pressure field map was limited by the test system capacity. Further investigation and testing will be performed to extend the map toward the choke line, to study instabilities at speeds above $74 \mathrm{krpm}$ and to understand observed surge line discontinuity. This will include boosting refrigeration capacity, installation of an expanded range flowmeter in an attempt to run at higher flowrates and installation of a fiber-optic proximity sensor to measure shaft movement.

Testing showed that the motor casing pressure operates subatmospheric for conditions typically found in the Tevatron. Added procedures have been implemented to guard against contaminants and prevent locked rotor conditions.

We saw no evidence of increased rotor vibrations beyond normal levels as the compressor nears the surge point. The available equipment limited our spectrum results to average spectrum calculations. In the future, we want equipment to provide peak-hold spectrum analysis capabilities to more easily see critical speed resonance during start/stop or other transient conditions.

\section{ACKNOWLEDGMENT}

The authors are grateful to all those in the Cryogenic and Controls Departments who have contributed to the design and installation of the cryogenic test facility and data acquisition system used for this study. Fermilab is operated by Universities Research Association Inc. under Contract No. DE-AC02-76CH03000 with the United States Department of Energy.

\section{REFERENCES}

1. Theilacker, J. C., Cryogenics 34, pp. 107-110 (1994).

2. Chen, H. M., Howarth, R., Geren, B., Theilacker, J. C., and Soyars, W. M. "Application of Foil Bearings to Helium Turbocompressor", in Proceedings of the Turbomachinery Symposium 30, edited by J. Burnett, Turbomachinery Laboratory at Texas A\&M University, College Station, Texas, 2001, pp. 103-114.

3. Geren, B.F. and Higgins, M. T., Turbotechnology Services Corporation, private communication. 
4. Gistau, G. M., et al., "The $300 \mathrm{~W}-1.75$ K Tore Supra Refrigerator Cold Centrifugal Compressors Report," in Advances in Cryogenic Engineering 33, edited by R. W. Fast, Plenum Press, New York, 1988, pp. 675-681.

5. Gistau, G. M., et al., "Application Range of Cryogenic Centrifugal Compressors," in Advances in Cryogenic Engineering 35, edited by R. W. Fast, Plenum Press, New York, 1990, pp. 1031-1037.

6. Fuerst, J. D., "Selection of Cold Compressors for the Fermilab Tevatron", ," in Advances in Cryogenic Engineering 39, edited by P. Kittel, Plenum Press, New York, 1994, pp. 863-869.

7. Norris, B. L., Cryogenics 34, pp. 73-76 (1994). 